\title{
Dynamic Changes in Chest CT Images Over 167 Days in 11 Patients with COVID-19: A Case Series and Literature Review
}

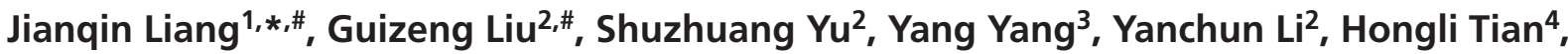 \\ Zhe Chen ${ }^{2}$ and Wenping Gong ${ }^{1, *}$
}

\begin{abstract}
Background: Recently, CT findings have been widely reported to be associated with the clinical severity of COVID-19. However, few studies have reported the correlation between CT findings and long-term outcomes in patients with COVID-19.

Case presentation: Herein, we conducted a 167 day long-term follow-up of CT examination on 11 patients with COVID-19 to evaluate their long-term prognosis, particularly in severe cases. We found that the course of COVID-19 can be divided into four stages according to the characteristics of CT images: 1) early stage (1-4 days), with chest CT showing quasi-circular ground-glass shadows and fine mesh shadows; 2 ) progressive stage (5-10 days), showing lesion spread through the axial interstitium along the bronchi and gradual diffusion to the whole lung; 3 ) recovery stage (11-74 days), showing gradual absorption of the fibre cord, ground-glass, and consolidation shadows; and 4) normal stage (74 days later), indicating no serious permanent lung injuries.

Conclusions: Our data indicate that chest CT can enable early detection of COVID-19 and determination of the different stages of COVID-19. Furthermore, mild cases tended to have better prognosis, whereas severe cases still showed cord-like fibrosis in the lungs in follow-up at the 167th day after symptom onset.
\end{abstract}

Key words: COVID-19, Chest CT, Long-term follow up, Prognosis, Case report

\section{BACKGROUND}

The diagnosis of coronavirus disease 2019 (COVID-19) is mainly based on positive nucleic acid findings of the novel coronavirus by real-time fluorescent reverse transcription polymerase chain reaction (RT-PCR), observation of a viral gene sequence highly homologous to the known novel coronavirus, or positive expression of specific immunoglobulin M (IgM) and IgG antibodies [1]. However, some patients' pulmonary imaging findings precede their clinical symptoms, and different imaging characteristics are seen in different periods. In the "Guidelines for Diagnosis and Treatment of COVID-19 (Trial Version 5 )" in China, cases of suspected pneumonia identified through imaging findings in Hubei Province are listed as clinically confirmed cases [2]. Therefore, imaging-based diagnosis is essential in guiding early isolation and treatment. Previous studies have suggested that typical chest CT features can be used to determine the severity of
Edited by:

Jian Wu, State Key Laboratory for Diagnosis and Treatment of Infectious Diseases, National Clinical Research Center for Infectious Diseases, The First Affiliated Hospital, Zhejiang University School of Medicine, China

Reviewed by:

Jun Cheng, Department of Clinical Research, The 903rd Hospital of PLA, China

Haicheng Tang, Department of Respiratory Medicine, Shanghai Public Health Clinical Center, Fudan University, China

\section{*Corresponding author:}

E-mail: gwp891015@whu.edu.cn,

Tel: +86-010-6677-5675 (WG)

E-mail: ljabj309@163.com,

Tel: +86-010-6677-5967 (JL)

${ }^{1}$ Tuberculosis Prevention and Control Key Laboratory/Beijing Key Laboratory of New Techniques of Tuberculosis Diagnosis and Treatment, Senior Department of Tuberculosis, The Eighth Medical Center of PLA General Hospital, \#17 Road Heishanhu, Haidian District, Beijing 100091, China ${ }^{2}$ Computer Tomography (CT) Radiology Department, Nanpi County People's Hospital, Cangzhou, Hebei 061500, China

${ }^{3}$ Medical Education Department, Nanpi County People's Hospital, Cangzhou, Hebei 061500, China ${ }^{4}$ Respiratory and Infectious Disease Department, Nanpi County People's Hospital, Cangzhou, Hebei 061500, China

\#Jianqin Liang and Guizeng Liu contributed equally to this article and were considered co-first authors.

Received: July 162021

Revised: August 52021

Accepted: August 82021

Published Online: August 262021 
COVID-19 and to predict COVID-19 clinical progress [3-6]. A recent study published in the Lancet on January 9, 2021, has evaluated the long-term outcomes of patients with COVID-19 [7] and found that 76\% of patients still have at least one persistent symptom 6 months after onset. The most common symptoms are fatigue, muscle weakness, sleep disorders, anxiety, and depression. However, the correlation between CT findings and long-term outcomes in patients with COVID-19 remained unclear. Herein, we performed a 167 day long-term follow-up of CT findings in 11 patients with COVID-19 to evaluate the long-term prognosis.

\section{CASE PRESENTATION}

\section{Patient information}

Eleven patients (five males and six females, 7-80 years of age, with a mean age \pm standard deviation (SD) of $50.09 \pm$ 20.94 years; Table 1) with COVID-19 were enrolled at our hospital between January 16, 2020, and February 16, 2020. This study followed the Declaration of Helsinki guidelines and was approved by the Institutional Review Board of our hospitals. Written informed consent was obtained from all patients involved before enrolment.

The patients meeting the baseline criteria of the "Guidelines for Diagnosis and Treatment of Coronavirus Disease 2019 (COVID-19) (TrialVersion 7)" were included in this study [2]. The Chinese Centre for Disease Control and Prevention performed viral detection of nasopharyngeal swabs for all patients. The patients were negative for influenza $A$ virus ribonucleic acid (RNA), influenza $B$ virus RNA, and adenovirus deoxyribonucleic acid, and positive for severe acute respiratory syndrome coronavirus-2 (SARSCoV-2) RNA. On the basis of relevant epidemiological investigations, clinical manifestations, and nucleic acid test results, the pathogen was identified as SARS-CoV-2 by the Centre for Disease Control and Prevention, and the patients were confirmed to be infected with SARS-CoV-2.

The patients with COVID-19 were divided into four groups: mild, ordinary, severe, and critical, according to the "Diagnostic and Treatment Protocol for Novel Coronavirus Pneumonia" released by the Chinese government [2]. Furthermore, the imaging process for COVID-19 was divided into four phases according to the "Guide for Imaging Diagnosis of Novel Coronavirus-infected Pneumonia" [8]: early, progressive, critical, and recovery. This study combined the critical stage and the progressive stage because of the imaging changes that occurred mainly in the progressive stage.

Cases $6,7,8$, and 9 are families, and cases 10 and 11 are families (Fig 1). By back-tracing the patients' trajectories, we found that patient 1 had a history of travel to Wuhan. The remaining ten people had a history of close contact with people diagnosed with COVID-19. Unfortunately, case 1 and case 6 died because of old age and complications of underlying diseases on April 7,2020. The other nine patients were cured (Table 1).

Several therapeutic measures were provided to the patients, including antiviral, anti-inflammatory, symptomatic treatment, and treatment with traditional Chinese medicine. Patients were considered cured if they met all the following criteria: (1) disappearance of clinical symptoms and signs; (2) return of complete blood count and biochemical blood index to normal; (3) two negative nucleic acid tests of nasopharyngeal swab or sputum samples, performed with RT-PCR (with sampling times at least 24 hours apart); (4) blood oxygen saturation in a non-oxygenated state $>93 \%$; and (5) chest CT showing no abnormal or residual fine fibre cord shadows.

\section{Clinical features and preliminary laboratory tests}

The clinical features and laboratory results of these 11 patients are listed in Table 1. Among the 11 cases, ten (90.91\%) began with fever. Case 11 involved a 7-year-old child without any symptoms causing discomfort, such as fever (maximum body temperature of $36.3{ }^{\circ} \mathrm{C}$ ). Because her mother had confirmed COVID-19, the child was routinely examined with nasopharyngeal swab tests twice. Both tests were positive, and chest CT indicated lesions in the lung. On the basis of these results, the girl was confirmed to have COVID-19. In contrast, the other ten patients had fever with body temperatures between $37.6^{\circ} \mathrm{C}$ and $39^{\circ} \mathrm{C}$ (mean value $37.83 \pm 0.64{ }^{\circ} \mathrm{C}$ ). The duration of fever in these patients ranged from 2 hours to 12 days. The incubation period for the 11 patients ranged from 7 to 20 days, with an average of $10.45 \pm 3.75$ days. Furthermore, the ten patients had other nonspecific clinical manifestations, such as dry cough, weakness, aches, and discomfort.

To determine the differences in the preliminary laboratory test results, we divided the 11 patients into three groups: ordinary, severe, and critical. The preliminary laboratory data for each patient in the most severe condition and receiving oxygen were obtained and compared, such as white blood cell (WBC) count $\left(\times 10^{9} / \mathrm{L}\right)$, lymphocyte count $\left(\times 10^{9} / \mathrm{L}\right)$, C-reactive protein $(\mathrm{CRP} ; \mathrm{mg} / \mathrm{L})$, respiratory rate (RR; times/min), $\mathrm{PO}_{2}(\mathrm{mmHg}), \mathrm{PCO}_{2}(\mathrm{mmHg})$, potential of Hydrogen (PH), $\mathrm{PaO}_{2}(\%)$, and $\mathrm{PaO}_{2} / \mathrm{FiO}_{2}$. No significant differences were observed among groups (Fig S1). Interestingly, compared with those in cured patients, the lymphocyte counts (Fig 2A, $P=0.0315$ ) and $\mathrm{PCO}_{2}$ (Fig 2B, $P=0.0494)$ were significantly lower in deceased patients, but the value of RR (Fig 2C, $P=0.0065$ ) was significantly higher. No differences were found in CRP (Fig 2D), $\mathrm{PO}_{2}$ (Fig 2E), WBC count (Fig 2F), $\mathrm{PH}$ (Fig 2G), $\mathrm{PaO}_{2}$ (Fig 2H), and $\mathrm{PaO}_{2} / \mathrm{FiO}_{2}$ (Fig 2I) between the cured and dead groups.

\section{Chest CT features}

We summarized the lesion properties in the progressive stage on chest CT among the 11 cases. The lesions were diverse in nature: eight patients $(72.73 \%)$ had ground-glass shadows, four patients (36.36\%) had nodular shadows accompanied by halo signs, four patients (36.36\%) had fine mesh shadows, four patients $(36.36 \%)$ had consolidation combined with ground-glass shadows, two patients (18.18\%) had consolidation accompanied by air bronchogram signs, and two patients $(18.18 \%)$ had reversed halo signs. 


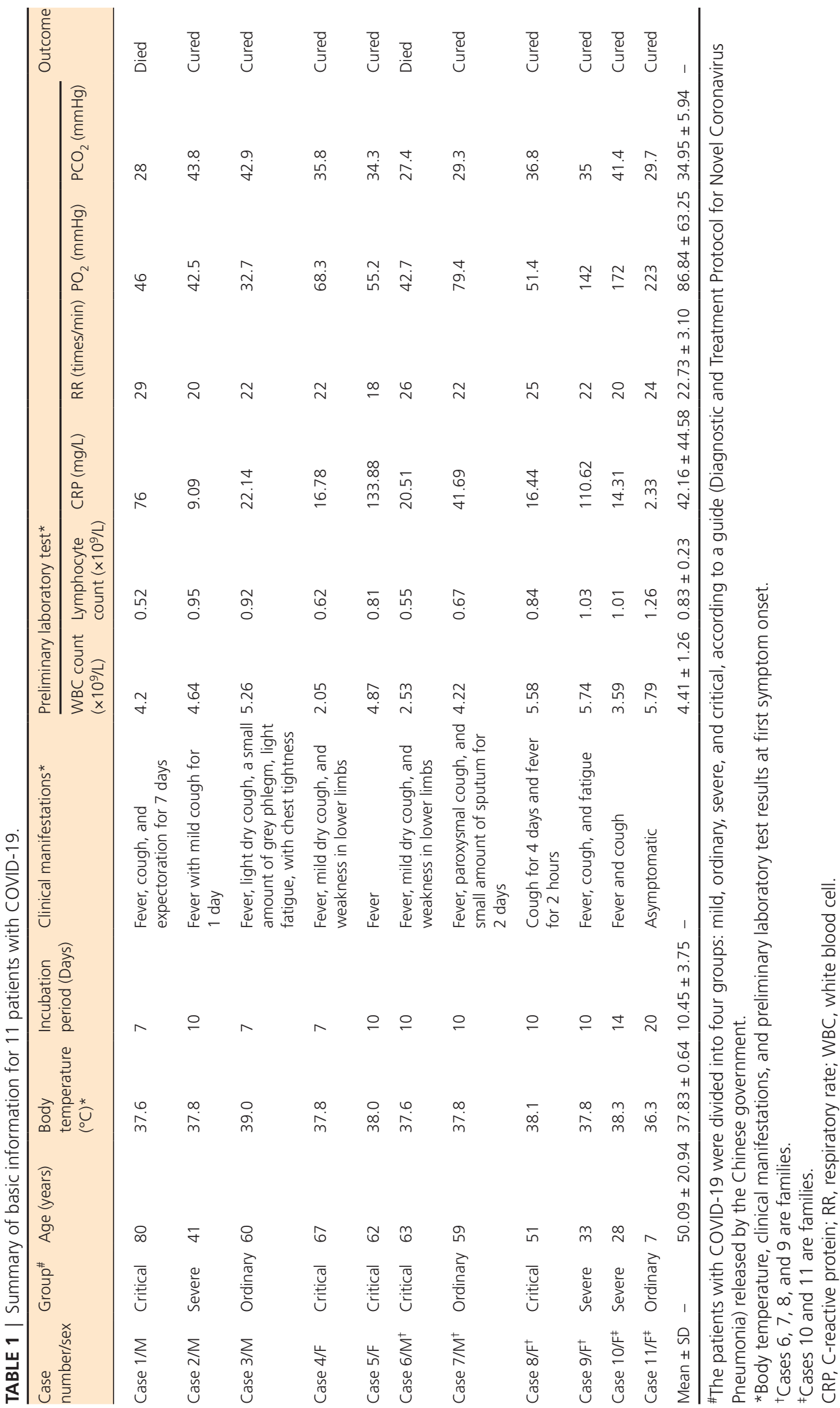




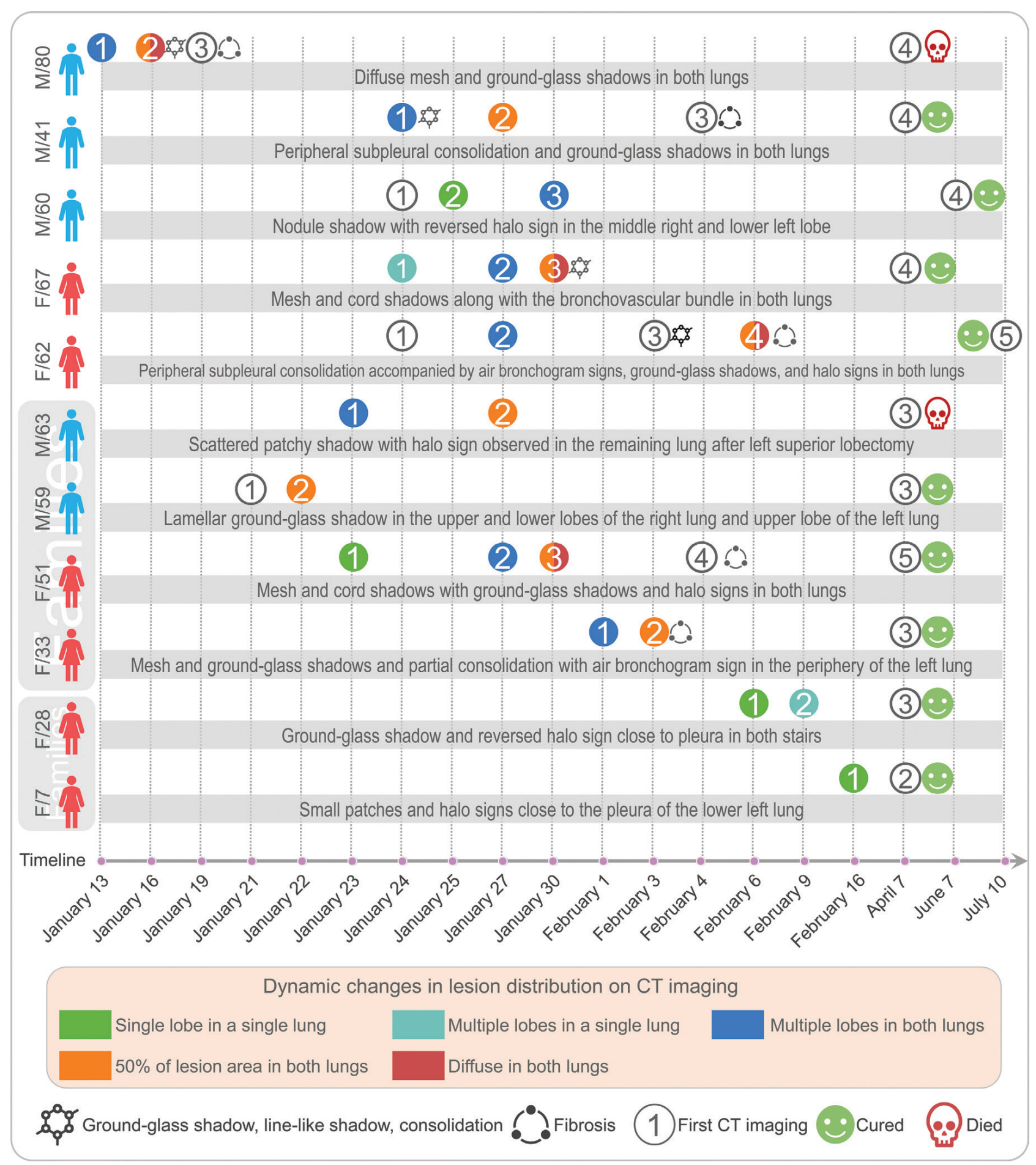

FIGURE 1 | The characteristics and dynamic changes in chest CT images over 167 days.

The lesions had different locations: unilateral pulmonary changes were observed in two cases (18.18\%), and bilateral pulmonary changes were observed in nine cases $(81.82 \%)$. Single lobe lesions were found in one case $(9.09 \%)$, and multilobar lesions were found in ten cases $(90.91 \%)$, including lesions in five lobes in four cases (40\%), lesions in two lobes in three cases (30\%), lesions in three lobes in two cases $(20 \%)$, and lesions in four lobes in one case $(10 \%)$. More lesions were located at the lung periphery close to the pleura, and gradually spread to the whole lung. No pleural effusion, cavitation, or lymphadenoma were observed among all patients (Fig 1).

\section{CT findings in the early stage}

The period from 1 to 4 days after the initial symptoms' onset was considered the early stage in our study. Among the 11 cases, 8 cases $(72.73 \%)$ showed a quasi-circular irregular patchy ground-glass density shadow (Fig 3). 
A

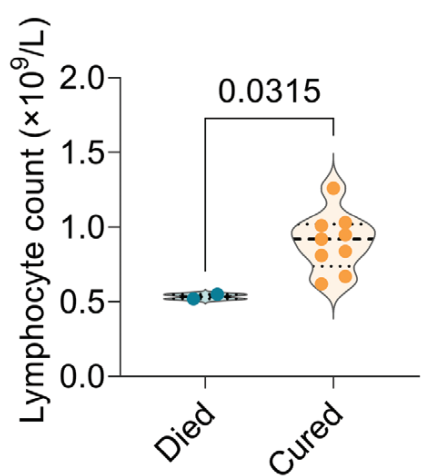

D

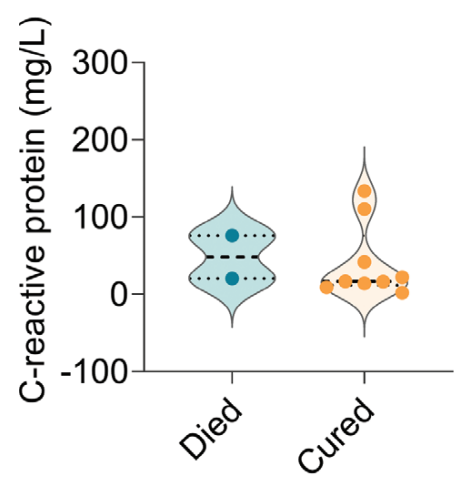

G

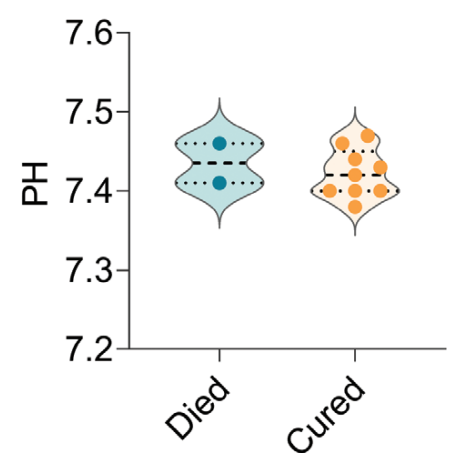

B

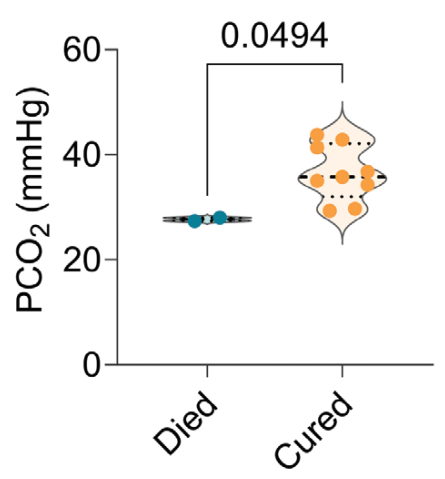

E

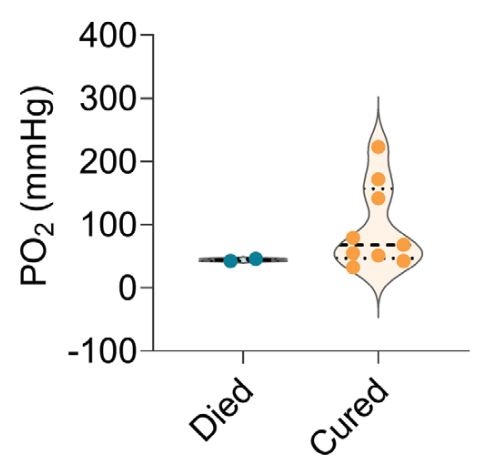

H

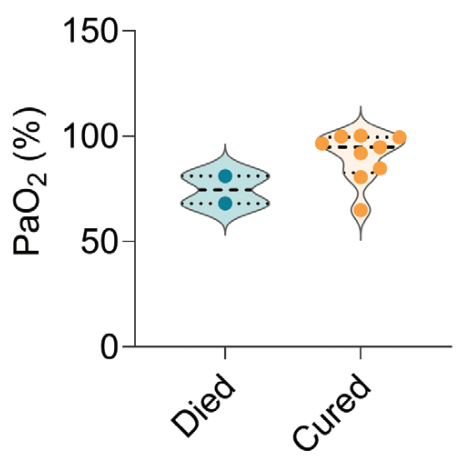

C

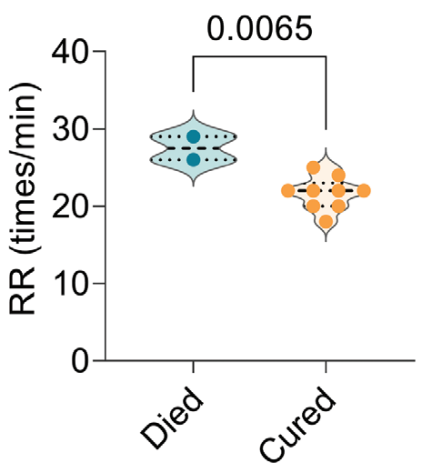

F

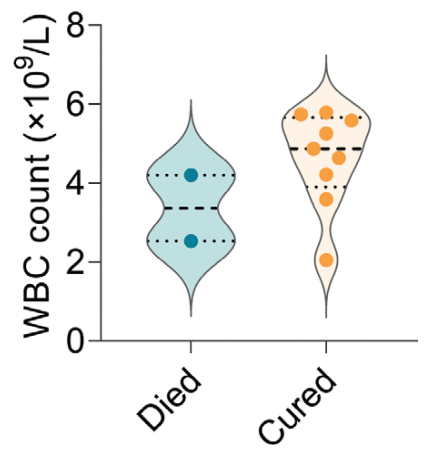

I

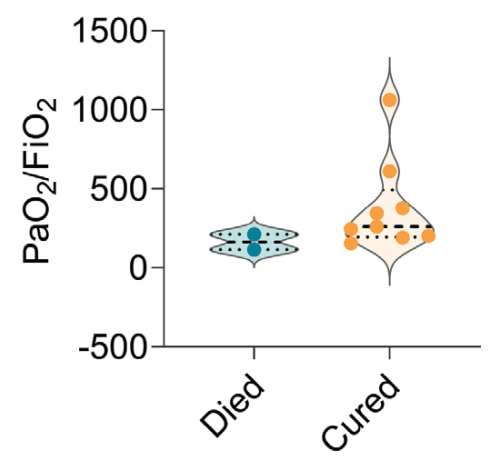

FIGURE 2 Preliminary laboratory test results between patients who died and were cured. The lymphocyte count $\left(\times 10^{9} / \mathrm{L}, \mathrm{A}\right), \mathrm{PCO},(\mathrm{mmHg}$, B), RR (times/min, C), CRP (mg/L, D), $\mathrm{PO}_{2}$ (mmHg, E), WBC count $\left(\times 10^{9} / \mathrm{L}, \mathrm{F}\right), \mathrm{PH}(\mathrm{G}), \mathrm{PaO}_{2}(\mathrm{H})$, and $\mathrm{PaO}_{2} / \mathrm{FiO}_{2}$ (I) between patients who died and were cured were compared with unpaired t-test or nonparametric Mann Whitney test according to the data normality. All data are shown as mean + SD $(n=2$ or 9 in patients who died or were cured, respectively). $P<0.05$ was considered significantly different.

Five cases $(45.45 \%)$ showed a small lamellar shadow in a single lobe in a single lung (Fig 3A-C, and E). One case showed multilobar lesions in both lungs, accompanied by delicate mesh shadows and consolidation (Fig 3D). Case 11 was a 7-year-old child with a small flaky coalition accompanied by a halo sign of the peripheral ground-glass cloud (Fig 3F). Interestingly, we found that CT findings of the children's images were different from these of adults and were characterized by inconspicuous interstitial lesions.

\section{CT findings in the progressive stage}

The period 5-10 days after initial symptom onset was considered the progressive stage in our study. An enlarged and fused lesion range, multiple lobular foci, and thickened pulmonary interstitium and interlobular septum were mainly accompanied by ground-glass shadows and fine mesh shadows. Among the 11 cases, $50 \%$ of the lesion area developed in both lungs within 3 days in four cases, within 1 week in two cases, and within 12 days in one case. Cord shadows and lesion consolidation gradually appeared, and halo signs accompanied some ground-glass shadows. Most of the lesions spread along the bronchus axial interstitium and progressively diffused to the entire lung, with air bronchograms, paving stone signs, halo signs, and reversed halo signs. No thickened bronchial wall or occluded lumen was observed. For critical cases, a diffuse 

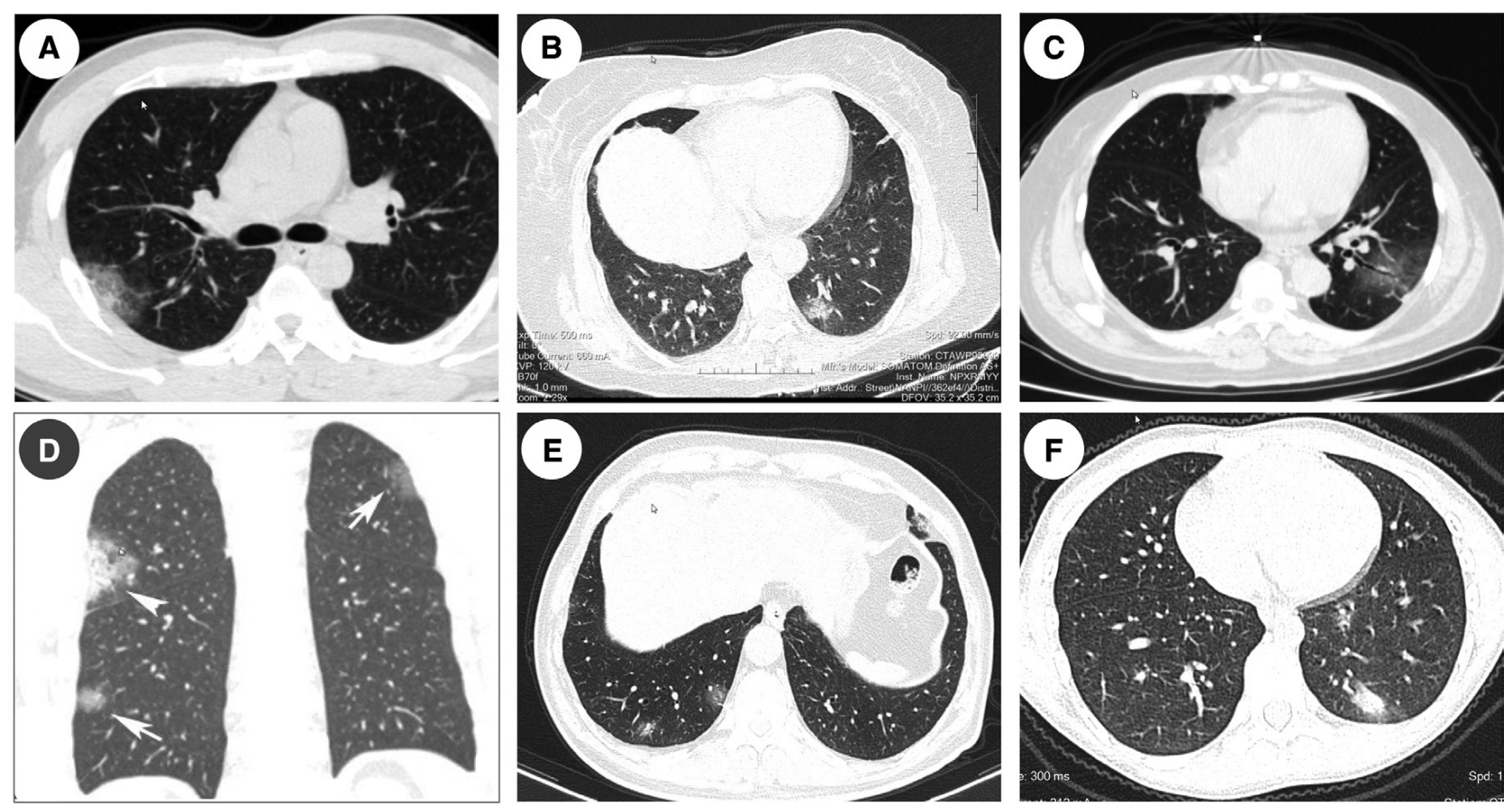

FIGURE 3 | Chest CT findings in the early stage in six cases. (A) Male, 41 years old, with a patchy ground-glass shadow in a single lobe of a single lung; (B) Female, 62 years old, with a quasi-circular ground-glass shadow in a single lobe of a single lung. (C) Female, 55 years old, with a patchy ground-glass shadow in a single lobe of a single lung. (D) Male, 60 years old, with multilobar patchy ground-glass shadows in both lungs. (E) Male, 59 years old, with a multifocal round ground-glass shadow in a single lobe of a single lung. (F) Female, 7 years old, with a small multifocal round ground-glass shadow in a single lobe of a single lung and consolidation in the middle part of the posterior basal segment lesion, surrounded by a halo sign of a ground-glass shadow.

fine mesh shadow was seen in both lungs, presenting as a 'white lung' sign (Fig 4).

\section{CT findings in the recovery stage}

The recovery stage began on day 11 after the onset of initial symptoms. For some patients, the images began to indicate that the lesions had been absorbed, and the range of pulmonary lesions was limited from the 11th day after symptom onset, mainly with fibre cords and light ground-glass shadows. The most apparent manifestations were observed from 14 to 21 days. Subsequently, we observed further absorption of the fibre cord and ground-glass shadows. Although the pulmonary lesions in mild cases showed good recovery, the pulmonary cord fibrosis persisted in severe cases (case 5, Fig 5) and were observed the 167th day after the onset of the disease, thus indicating that the recovery of the lung in severe cases of COVID-19 is slower than that in mild cases. Our results may provide evidence supporting the conclusions of the recently published study by Huang et al. [7].

\section{DISCUSSION}

Since December 2019, COVID-19 outbreaks have occurred in many parts of the world, and the number of confirmed cases and deaths has rapidly increased. This disease poses a major threat to human life and health [9-12]. COVID-19 is mainly characterized by respiratory tract infection and is easily transmissible among people. Up to $86 \%$ of infected patients develop pneumonia, and some progress to severe pneumonia or even death $[13,14]$. Because radiographic changes preceded clinical symptoms in some COVID-19 cases, chest CT features and their dynamic changes in patients with COVID-19 were found to be conducive to the early detection of the infection source, early isolation and intervention, and prognosis prediction.

In this study, case 11 was an asymptomatic confirmed case. This patient's early chest CT findings showed consolidation of the nodules with halo signs (Fig 3F) that slowly progressed, in contrast to the early chest CT findings seen in adults, which showed fine mesh and ground-glass shadows as the interstitial changes rapidly progressed after 3 to 4 days. The dynamic changes in the chest CT imaging characteristics of COVID-19 can be summarized as follows. The early stage is 1-4 days after the onset of the initial symptoms. Most commonly, the lesions are limited to peripheral subpleural lesions in a single lobe of a single lung (Figs 3A, B, $\mathrm{C}, \mathrm{E}, 5 \mathrm{~A}, 5 \mathrm{~B}$ ) and multilobar lesions in both lungs (Fig 3D), in agreement with findings from previous reports $[15,16]$. The lesions mainly show interstitial infiltration, and $72.73 \%$ $(8 / 11)$ are seen as quasi-circular ground-glass shadows, owing to the increased density of the lung caused by alveolar swelling, a small amount of alveolar exudation, and alveolar septal inflammation [17].

The progressive stage occurs 5-10 days after the onset of the initial symptoms: the lesions are enlarged and develop into multilobar lesions in a single lung (18.18\%) or multilobar lesions in both lungs (81.82\%) (Figs 4A, 4B, 4C, 5C). A previous study has indicated that lesions progress from 

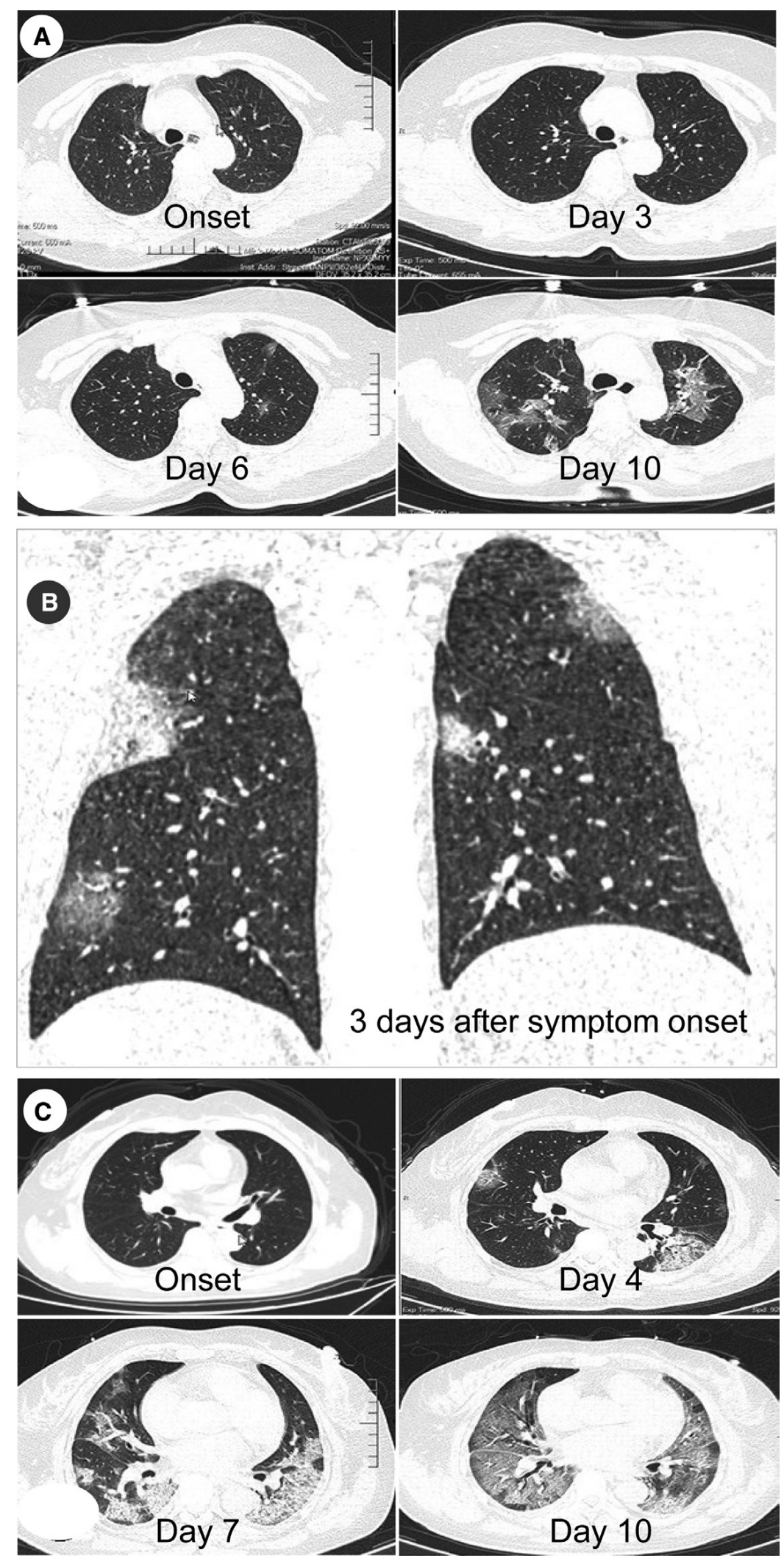

FIGURE 4 | Chest CT findings in the progressive stage in three cases. (A) Female, 62 years old, examined at symptom onset and on days 3, 6 , and 10; the lesion gradually enlarged with ground-glass shadows and a delicate mesh of interstitial shadows. In addition, consolidation and cord shadows gradually appeared. (B) Male, 41 years old, examined 3 days after symptom onset; multilobar lesions in both lungs with groundglass shadows and interstitial fine mesh shadows were observed. (C) Female, 55 years old, examined at symptom onset and on days 4, 7, and 10; the early manifestations were single-lobe ground-glass lesions in a single lung. The range gradually increased after 4 days, with multilobar lesions in both lungs, presenting as ground-glass shadows and fine mesh shadows. After day 7 , the range further increased to more than $50 \%$ of the lesion area in both lungs. After day 10, diffuse ground-glass shadows and interstitial fine mesh shadows were observed in both lungs. 


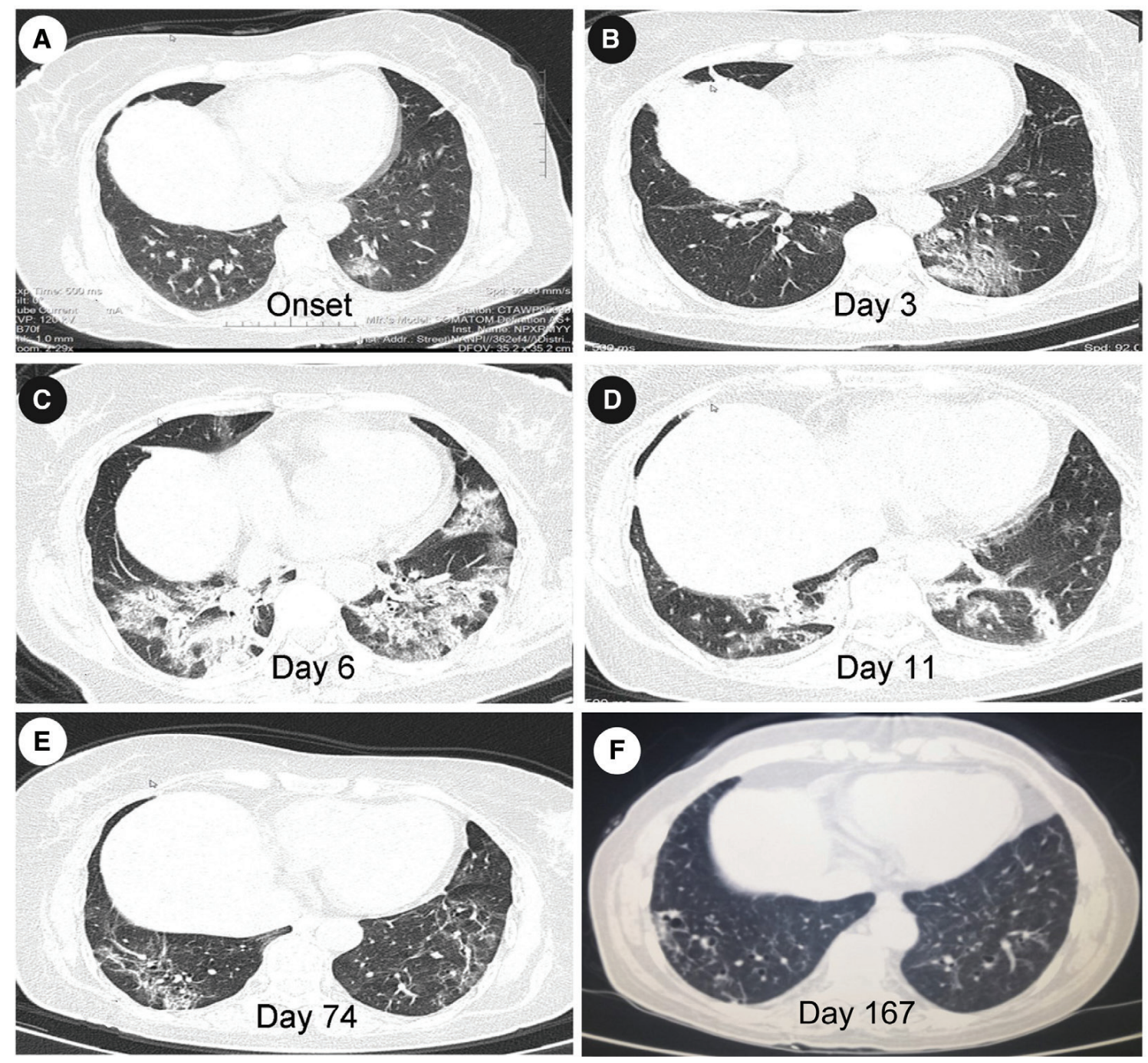

FIGURE 5 | The entire progression of chest CT findings of case 5 from early stage, progressive stage, recovery stage to normal stage. (A) At the time of symptom onset, a ground ground-glass shadow was observed in a single lobe of a single lung. (B) On day 3 , the lesion was enlarged, with ground-glass shadows and interstitial fine mesh shadows. (C) On day 6, the lesion was further enlarged, presenting multiple lesions in both lungs, with ground-glass shadows and thick interstitial shadows. (D) On day 11, the lesion began to shrink, mainly with coarse fibre cords. (E) On day 74 of follow-up, the lesion was stable, and cord-like fibrosis was observed. (F) On the 167th day of follow-up, the lung lesions were further alleviated, but cord-like fibrosis was still seen.

the periphery to the central area [8]. The lesions primarily showed interstitial infiltration, and four cases (36.36\%) had ground-glass shadows accompanied by consolidation (Fig 4B), an air bronchogram sign (Fig 4C), a paving stone sign (Fig 4A), or a halo sign (Fig 4B), as reported in the literature [14-18]. A patient with acute disease progressed to adult respiratory distress syndrome, with significantly decreased blood oxygen levels, and had CT features of high-density consolidation and diffuse mesh shadows (white lung) in both lungs (Fig 4C), as reported in the literature $[14,18]$. The recovery stage started 11 days after the onset of the initial symptoms: except for two patients $(18.18 \%)$ who died from underlying diseases, nine patients $(81.82 \%)$ had a decreased distribution range of lesions within 2-3 weeks after symptom onset. Severe and critical cases showed gradually sparse mesh shadows in both lungs (Fig 5D). After 3 weeks, the lesions were characterized by fibre cords (Fig 5E).
Jin et al. have divided the CT characteristics of COVID-19 into super-early, early, rapid progression, consolidation, and dissipation stages [19]. Super early refers to $1-2$ weeks after infection exposure, when no clinical symptoms are present, but CT reveals single or multiple subpleural lesions or nodular shadows with air-filled bronchi. Among the 11 confirmed cases, only the 7-year-old child showed these imaging features in the super-early stage (Fig $3 \mathrm{~F}$ ), and the lesions on chest $\mathrm{CT}$ did not develop into the progressive phase but instead directly developed into the recovery stage after active treatment; this patient was gradually cured.

The dynamic CT changes in the remaining ten cases followed the progression from the early phase (1-3 days of onset) to the progressive phase and consolidation (3-14 days of onset) reported by Pan et al. [18] and Jin et al. [19]. However, in this study, our patients entered the recovery period on the $11^{\text {th }}$ day after initial diagnosis, a time earlier 
than the 14-18 days reported in previous studies [18-21]. The reason for this difference may be that the cases studied by the above researchers were in Wuhan, Hubei Province. Because of the concentration of cases in Wuhan, the relative severity of the disease probably affected the timing. However, the inflammatory response was not severe, and the symptoms and CT changes were relatively mild. Similarly, a recent study by Wang et al. has reported that $75.0 \%$ of the patients rapidly progressed within 6-9 days, and $76.9 \%$ of the patients showed significantly recovered lesions within 10-14 days [22]. These data indicate that the dynamic CT changes in patients with COVID-19 in county-level hospitals in Hebei Province were slightly different from those in Hubei Province.

Wang et al. have reported that $94 \%$ of patients discharged after 1 month of treatment have residual lesions on the final CT scan, and ground-glass shadows are the most common feature [23]. We observed that fibrosis was rare in mild cases; fibrosis and ground-glass shadows were gradually absorbed in severe and critical cases; and chest CT showed sparse linear shadows, which had no effect on lung function and could be clinically cured. These features differed from the lung fibrosis and permanent lung injuries observed in SARS and Middle East respiratory syndrome infections [14]. Li et al. have reported a multicentre study examining CT changes in COVID-19 in southwest China [24]. With an observation time of 43 days, the authors did not study the correlation between disease course and imaging features. Pan et al. have discussed the correlation between the disease course and imaging features in 21 cases of COVID-19 from Wuhan and divided these cases into four stages [18]. With an observation time of 26 days, no follow-up was conducted for discharged patients. Our patients were followed up for 167 days until July 10, 2020. Except for the two patients who died on April 7, 2020, the remaining patients achieved clinical cure standards. Notably, this is the longest follow-up time reported in the current literature, and the findings also reflect the treatment level of COVID-19 in county-level hospitals in China.

However, this study has some limitations, as follows. (1) The number of samples was small, and only descriptive analyses were conducted on the imaging manifestations of COVID-19 in different stages. (2) There was only one paediatric case, which cannot fully represent the imaging characteristics among children. (3) No subgroup analyses of mild and severe cases were performed. (4) Because of policy regulations, complete laboratory test results of 11 patients could not be obtained.

\section{CONCLUSIONS}

The dynamic changes in the chest CT characteristics of COVID-19 were as follows. The early stage is 1-4 days after the onset of initial symptoms and is characterized by ground-glass lesions, which are more commonly observed around the subpleural lung than single lobes. The progressive stage is $5-10$ days after the onset of the initial symptoms, and its imaging manifestations are lesions spreading from the periphery to the centre, and showing consolidation and fusion. With active treatment, the recovery stage begins on day 11 after symptom onset, and the fibre cord, groundglass, and consolidation shadows are gradually absorbed. On the basis of pulmonary imaging, the recovery of lesions in mild cases of COVID-19 is relatively better, whereas that in severe cases is slower, and the shadow of cord-like fibrosis can still be seen on the 167th day after symptom onset. However, CT examination in the early diagnosis of COVID-19 should not be arbitrarily adopted but should be discussed in combination with the results of laboratory SARS-CoV-2 nucleic acid testing.

\section{ABBREVIATIONS}

COVID-19, coronavirus disease 2019

$\mathrm{CT}$, computer tomography

CRP, C-reactive protein

IgM, immunoglobulin $M$

PLA, People's Liberation Army

$R R$, respiratory rate

SARS-CoV-2, severe acute respiratory syndrome coronavirus-2

$\mathrm{SD}$, standard deviation

WBC, white blood cell

\section{REFERENCES}

1. Commission GOoNH, Medicine GOoNAoTC. [Diagnostic and treatment protocol for novel coronavirus pneumonia]. Trial version 7 ed. Beijing: General Office of National Health Commission; 2020.

2. Committee GOoNH, Medicine GOoNAoTC. [Diagnostic and treatment protocol for novel coronavirus pneumonia]. Trial version 7 ed. Beijing: General Office of National Health Committee; 2020.

3. Salahshour F, Mehrabinejad MM, Nassiri Toosi M, Gity M, Ghanaati $H$, Shakiba $M$, et al. Clinical and chest $C T$ features as a predictive tool for COVID-19 clinical progress: introducing a novel semi-quantitative scoring system. Eur Radiol. 2021:1-11.

4. Oliver SE, Gargano JW, Marin M, Wallace M, Curran KG, Chamberland $\mathrm{M}$, et al. The Advisory Committee on Immunization Practices' Interim Recommendation for Use of Moderna COVID-19 Vaccine - United States, December 2020. MMWR Morb Mortal Wkly Rep. 2021;69:1653-1656.

5. Lei Q, Li G, Ma X, Tian J, Wu YF, Chen H, et al. Correlation between $\mathrm{CT}$ findings and outcomes in 46 patients with coronavirus disease 2019. Sci Rep. 2021;11:1103.

6. Hashemi-Madani N, Emami Z, Janani L, Khamseh ME. Typical chest CT features can determine the severity of COVID-19: a systematic review and meta-analysis of the observational studies. Clin Imaging. 2021;74:67-75.

7. Huang C, Huang L, Wang Y, Li X, Ren L, Gu X, et al. 6-Month consequences of COVID-19 in patients discharged from hospital: a cohort study. Lancet. 2021;397:220-232.

8. Li H-J, Xu H-B. Guideline for imaging diagnosis of novel coronavirus (2019-ncov) infected pneumonia (1st edition 2020). New Med. 2020;30:22-34.

9. Jia Z, Gong W. Will mutations in the spike protein of SARSCoV-2 lead to the failure of COVID-19 vaccines? J Korean Med Sci. 2021;36:e124.

10. Gong W, Wu X. Is the tuberculosis vaccine BCG an alternative weapon for developing countries to defeat COVID-19? Indian J Tuberc. 2021;68:401-404.

11. Gong W, Aspatwar A, Wang S, Parkkila S, Wu X. COVID-19 pandemic: SARS-CoV-2 specific vaccines and challenges, 
protection via BCG trained immunity, and clinical trials. Expert Rev Vaccines. 2021:1-24.

12. Aspatwar A, Gong W, Wang S, Wu X, Parkkila S. Tuberculosis vaccine $B C G$ : the magical effect of the old vaccine in the fight against the COVID-19 pandemic. Int Rev Immunol. 2021; Published online first: 1-14.

13. Chan JF, Yuan S, Kok KH, To KK, Chu H, Yang J, et al. A familial cluster of pneumonia associated with the 2019 novel coronavirus indicating person-to-person transmission: a study of a family cluster. Lancet. 2020;395:514-523.

14. Hosseiny M, Kooraki S, Gholamrezanezhad A, Reddy S, Myers L. Radiology perspective of coronavirus disease 2019 (COVID-19): lessons from severe acute respiratory syndrome and middle east respiratory syndrome. AJR Am J Roentgenol. 2020;214:1078-1082.

15. Chung $M$, Bernheim A, Mei X, Zhang N, Huang M, Zeng $X$, et al. CT imaging features of 2019 novel coronavirus (2019nCoV). Radiology. 2020;295:202-207.

16. Song F, Shi N, Shan F, Zhang Z, Shen J, Lu H, et al. Emerging 2019 novel coronavirus (2019-nCoV) pneumonia. Radiology. 2020;295:210-217.

17. Koo HJ, Lim S, Choe J, Choi SH, Sung H, Do KH. Radiographic and $\mathrm{CT}$ features of viral pneumonia. radiographics: a review publication of the Radiological Society of North America, Inc. 2018;38:719-739.
18. Pan F, Ye T, Sun P, Gui S, Liang B, Li L, et al. Time course of lung changes at chest $C T$ during recovery from coronavirus disease 2019 (COVID-19). Radiology. 2020;295:715-721.

19. Jin YH, Cai L, Cheng ZS, Cheng H, Deng T, Fan YP, et al. A rapid advice guideline for the diagnosis and treatment of 2019 novel coronavirus (2019-nCoV) infected pneumonia (standard version). Mil Med Res. 2020;7:4

20. Xiong Y, Sun D, Liu Y, Fan Y, Zhao L, Li X, et al. Clinical and high-resolution CT features of the COVID-19 infection: comparison of the initial and follow-up changes. Invest Radiol. 2020;55:332-339.

21. Shi H, Han X, Jiang N, Cao Y, Alwalid O, Gu J, et al. Radiological findings from 81 patients with COVID-19 pneumonia in Wuhan, China: a descriptive study. Lancet Infect Dis. 2020;20:425-434.

22. Wang J, Liu J, Wang Y, Liu W, Chen X, Sun C, et al. [Dynamic changes of chest $C T$ imaging in patients with corona virus disease-19 (COVID-19)]. Zhejiang Da Xue Xue Bao Yi Xue Ban. 2020;49:191-197.

23. Wang $Y$, Dong $C$, Hu Y, Li C, Ren Q, Zhang $X$, et al. Temporal changes of $C T$ findings in 90 patients with COVID-19 pneumonia: a longitudinal study. Radiology. 2020;296:E55-E64.

24. Li X, Zeng W, Li X, Chen H, Shi L, Li X, et al. CT imaging changes of corona virus disease 2019 (COVID-19): a multicenter study in Southwest China. J Transl Med. 2020;18:154. 


\section{Supplementary material}
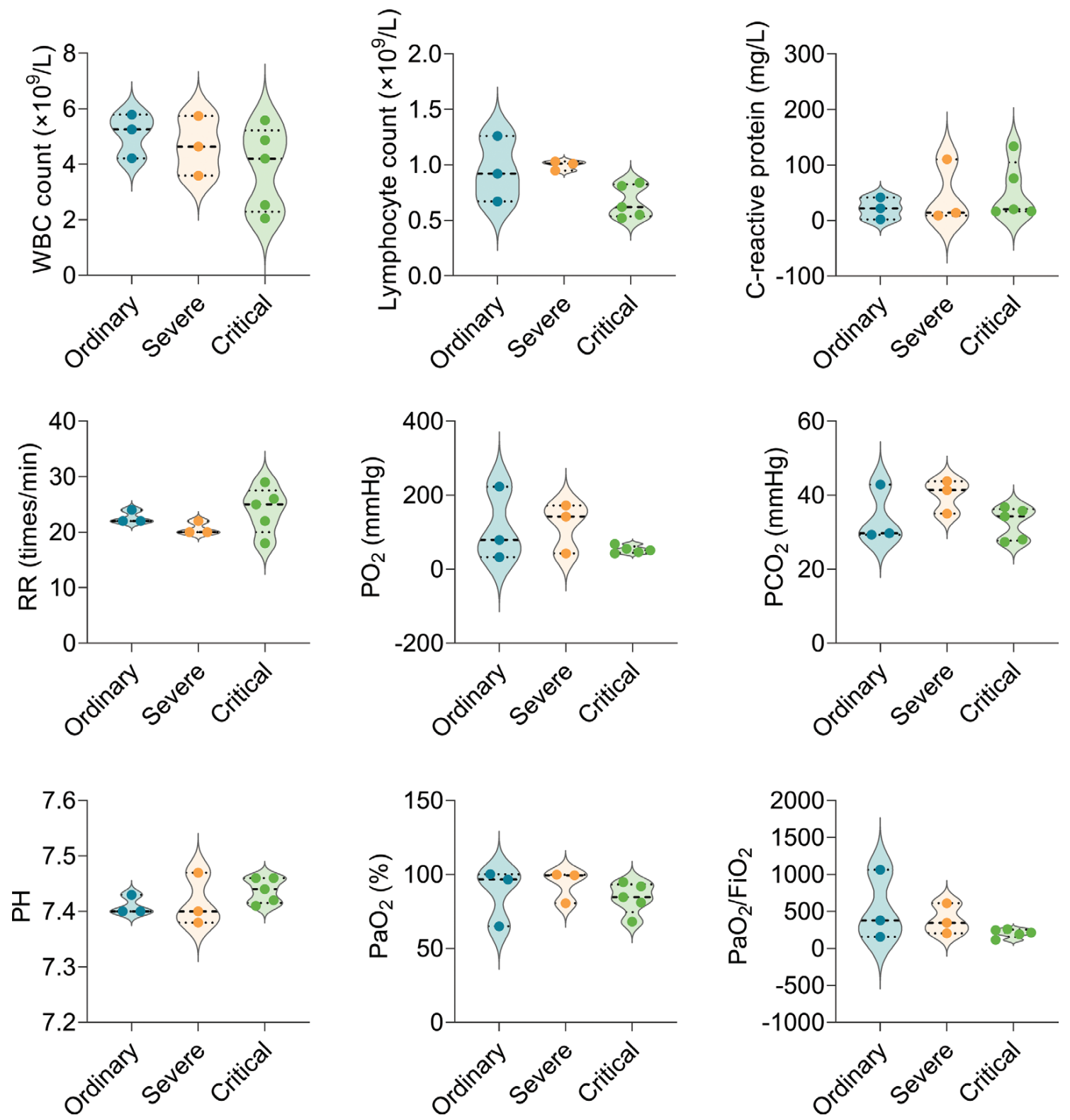

FIGURE S1 | Eleven patients were divided into three groups (ordinary, severe, and critical) according to the Diagnostic and Treatment Protocol for Novel Coronavirus Pneumonia. The WBC count ( $\left.\times 10^{9} / \mathrm{L}\right)$, lymphocyte count $\left(\times 10^{9} / \mathrm{L}\right), \mathrm{CRP}(\mathrm{mg} / \mathrm{L}), \mathrm{RR}(\mathrm{times} / \mathrm{min}), \mathrm{PO}_{2}(\mathrm{mmHg})$, $\mathrm{PCO}_{2}(\mathrm{mmHg}), \mathrm{PH}, \mathrm{PaO}_{2}$, and $\mathrm{PaO}_{2} / \mathrm{FiO}_{2}$ among three groups were compared with one-way analysis of variance or Kruskal-Wallis test according to the data normality homogeneity of variances. All data are shown as mean $+\mathrm{SD}(n=3-5)$. $P<0.05$ was considered significantly different. 